\title{
New immunosuppressive drugs and lung transplantation: last or least?
}

\author{
J W K van den Berg, D S Postma, G H Koëter, W van der Bij
}

Lung transplantation has become an accepted treatment modality for end stage lung disease. ${ }^{1}$ Traditionally, immunosuppressive maintenance therapy consists of cyclosporin, azathioprine, and prednisolone in kidney and liver transplantation as well as in lung transplantation. Despite the use of these drugs, acute rejection occurs frequently, especially in the first weeks and months after lung transplantation. Although these periods are now almost never life threatening, they are associated with substantial morbidity. Prevalences of acute rejection ranging from $60 \%$ to $100 \%$ have been reported, depending on whether acute rejection is based on clinical or histological diagnosis. ${ }^{2-4}$ The incidence of acute rejection is far higher after lung transplantation than after any other form of solid organ transplantation. This may be due to the fact that the donor lung contains a substantial amount of immunocompetent tissue and because the lungs are constantly exposed to environmental factors. ${ }^{5}$

Bronchiolitis obliterans syndrome (BOS) is the major cause of morbidity and mortality in long term survivors of lung transplantation. ${ }^{6}{ }^{7}$ It occurs in approximately $30-50 \%$ of recipients still alive one year after transplantation and is characterised by progressive airway obstruction, usually in the presence of histological evidence of obliterative bronchiolitis (OB).$^{8}$ Once established it is usually refractory to immunosuppressive treatment, ${ }^{9}$ thus stressing the importance of preventing this long term complication. As the frequency and severity of periods of acute rejection are the main risk factors for development of $\mathrm{BOS},{ }^{8}$ it follows that it is of utmost importance to prevent acute rejection. A substantial effort has therefore been made to develop and introduce new immunosuppressant drugs, not only to prevent and treat acute and chronic rejection, but also with the hope of fewer side effects. This review will focus on recently approved immunosuppressant drugs, some of their pharmacological properties, evidence of their effectiveness in organ transplantation in general, and reported results in lung transplantation in particular. Furthermore, promising immunosuppressive drugs under investigation and the inhalation of cyclosporin or corticosteroids will be considered briefly.

\section{Mycophenolate mofetil}

In 1896 mycophenolic acid (MPA) was obtained from a species of Penicillium and its antifungal activity was noted. ${ }^{10}$ Mycophenolate mofetil (MMF, Cellcept ${ }^{\circledR}$ ) is the ester prodrug and is rapidly hydrolysed in vivo to MPA, the active compound. MPA strongly inhibits the enzyme inosine monophosphate dehydro- genase which is essential for the de novo purine synthesis in lymphocytes, thereby preventing lymphocyte proliferation. Bioavailability after oral administration of MMF is excellent, and it is renally excreted as an inactive salt after hepatic glucuronidation with a mean half life of approximately 18 hours. ${ }^{11}$ The recommended daily oral dosage is $1 \mathrm{~g}$ twice daily. Specific side effects are mainly gastrointestinal symptoms including nausea, diarrhoea, and cramps. The incidence of side effects has been shown to subside with longer use. ${ }^{12}$

Its efficacy in reducing the incidence of rejection after cadaveric renal transplantation has been investigated in three multicentre, randomised, double blind studies in which MMF was compared with either placebo or azathioprine. ${ }^{13-15}$ These large studies yielded comparable results. The incidence of rejection was about $50 \%$ lower in the groups treated with MMF than in the control groups. In a multicentre open label trial the efficacy and safety of MMF in combination with cyclosporin was compared with high dose steroids together with azathioprine and cyclosporin in patients with renal allograft rejection refractory to antilymphocyte globulin. Additional treatment with MMF resulted in a $45 \%$ reduction in graft loss and death six months after enrollment in the study. ${ }^{16}$ Zuckermann et al compared MMF with azathioprine in a non-randomised open trial in 40 lung transplant recipients (data presented at the European Society of Organ Transplantation, Budapest 1997, abstract 109) and found the incidence of histologically proven acute rejection in the group treated with MMF to be significantly lower six months after transplantation $(24 \%$ versus $87 \%)$. This study was hampered by a rather low survival rate at six months of $76 \%$ in the group treated with $\mathrm{MMF}$ and $65 \%$ in the azathioprine treated group. MMF has recently been compared with azathioprine in a non-randomised study in 22 lung transplant recipients. ${ }^{17} \mathrm{~A}$ lower incidence of biopsy proven rejection during treatment with MMF was found during the first 12 months after transplantation and the prevalence of BOS at one year in the azathioprine treated group was twice as high (36\%) as in the group treated with MMF (18\%), although this difference was not statistically significant. A European multicentre phase III trial is currently being conducted in which $\mathrm{MMF}$ is being compared with azathioprine after lung transplantation, both in conjunction with cyclosporin and corticosteroids. The primary end point is the development of BOS, with acute rejection being a secondary end point. 
Tacrolimus

Tacrolimus (FK506, Prograf $\AA$ ) is a macrolide antibiotic with a short history. It was isolated in 1986 from Streptomyces tsukubaensis. ${ }^{18} \mathrm{Al}-$ though structurally different from cyclosporin, tacrolimus has a similar mode of action. Both drugs bind to intracellular proteins and interfere with the signal transduction from $\mathrm{T}$ cell surface receptors to the nucleus in lymphocytes, preventing transcription of lymphokine genes involved in $\mathrm{T}$ cell activation.

Tacrolimus is a hydrophobic compound and bioavailability after oral administration is poor and variable. It is almost completely metabolised through the cytochrome P-450 pathway in the liver with a half life of about nine hours. Because of these pharmacokinetic properties and its narrow therapeutic index, drug level monitoring is essential. ${ }^{19}$

Both a large US study and a European multicentre trial have shown the superiority of tacrolimus based therapy over cyclosporin in reducing the incidence and severity of rejection up to one year after liver transplantation. No difference was found in terms of graft and patient survival. The tacrolimus based therapy was associated with more side effects, primarily nephrotoxicity and neurotoxicity. ${ }^{2021}$

In a prospective randomised study the efficacy of tacrolimus was compared with that of cyclosporin in 74 lung transplant recipients. Acute rejection occurred less frequently in the tacrolimus group (89\% versus $100 \%$ at six months), and fewer courses of methylprednisolone were given. ${ }^{4}$ Keenan et al reported the long term results of this study and found a markedly reduced incidence of biopsy proven $\mathrm{OB}$ in the tacrolimus treated group $(21.7 \%$ versus $38 \%$ ) with a trend towards improved survival. ${ }^{22}$ Tacrolimus has also been used as rescue therapy for BOS. In a non-randomised trial with 12 patients no improvement in lung function could be found although the rate of decline decreased. ${ }^{23}$ In a similar study in 10 single-lung transplant recipients with histologically confirmed $\mathrm{OB}$ the lung function stabilised after treatment was changed from azathioprine and cyclosporin to tacrolimus. ${ }^{24}$ Finally, in a retrospective study of 14 patients with recurrent or persistent acute rejection the incidence and severity of acute rejection declined significantly after maintenance immunosuppressive treatment was changed from cyclosporin to tacrolimus. ${ }^{25}$

\section{New drugs under investigation}

Sirolimus (rapamycin) is very similar to tacrolimus in being a product of a Streptomyces species and these drugs are structurally related. It binds to the same intracellular protein as tacrolimus, the so called FK-binding protein, yet it fails to inhibit the calcineurin phosphatase activity. Its effect depends on interfering with the cell cycle progression and it inhibits calcium independent signalling pathways in $\mathrm{T}$ and $\mathrm{B}$ cells. ${ }^{26}$ It appears that rapamycin not only inhibits the proliferation of lymphocytes, but of mesenchymal and endothelial cells also. ${ }^{27-29}$ It has subsequently been shown in animal airway transplant models that administration of rapamycin markedly inhibits the fibroproliferative response to transplantation. ${ }^{30}{ }^{31}$ As OB probably represents the result of this response to injury, it is hoped that the use of rapamycin in lung transplantation will lessen the impact of this devastating complication. Initial clinical experience from phase II trials in renal recipients suggests that the combination of rapamycin and cyclosporin is superior to cyclosporin alone in reducing the frequency of acute rejection episodes. ${ }^{32}$ Preliminary data from phase III trials of rapamycin in renal transplant recipients seem to confirm its efficacy, ${ }^{33}{ }^{34}$ but a remarkably high number of withdrawals from the study due to side effects was noted. A randomised controlled multicentre trial comparing rapamycin or a rapamycin derivative with azathioprine in patients at risk for BOS is likely to be conducted soon.

Specific antilymphocyte antibodies such as the polyclonal antithymocyte globulin and the monoclonal OKT3 have been used for some time in induction regimens or for the treatment of steroid resistant rejection. ${ }^{35}$ Their use is limited because of the increased risk of infection, troublesome side effects, and substantial costs. New blocking antibodies directed towards different cell receptors involved in the stimulation of alloreactive lymphocytes are currently being developed. From the results of two large randomised, placebo controlled trials it appears that the humanised anti-IL-2-receptor antibodies are effective in reducing the incidence of rejection after kidney transplantation. ${ }^{36} 37$

Mizoribine and brequinar sodium have much in common with MMF. They interfere with nucleotide biosynthesis, thereby inhibiting $\mathrm{B}$ and $\mathrm{T}$ cell proliferation. Mizoribine has been extensively used in Japan as a steroid sparing alternative to azathioprine. A European trial in kidney recipients showed that the incidence of rejection decreased with the use of mizoribine. $^{38}$ Brequinar sodium has not yet been used in human subjects.

\section{Inhalation of immunosuppressants}

Inhaled medication is commonly used in patients with asthma or chronic obstructive pulmonary disease as this route of administration allows a high dose of the drug to reach the affected organ with fewer systemic complications. The transplanted lung is, of course, also accessible to inhaled therapy. A lower incidence of $\mathrm{OB}$ was reported in seven heart-lung recipients treated with $2 \mathrm{mg}$ nebulised budenoside twice daily for acute rejection resistant to intravenous corticosteroids compared with a control group. ${ }^{39}$ Speich et $a l^{40}$ have described the effect of inhaled fluticasone in one patient with BOS grade II who was treated with ten treatment pairs of two weeks duration with either fluticasone $(1000 \mu \mathrm{g}$ twice a day) or placebo. Both subjective and objective improvement, as determined by lung function, occurred with high dose fluticasone. Iacono et al reported that seven out of nine patients with biopsy proven OB refractory to enhanced immunosuppression treated with aerosolised 
cyclosporin A had histological improvement and a reduced rate of decline in forced expiratory volume in one second $\left(\mathrm{FEV}_{1}\right)$ compared with pretreatment values and with nine untreated historical control recipients with $\mathrm{OB} .^{41}$ The same group reported similar results in patients with refractory acute allograft rejection. ${ }^{42} 43$

\section{Conclusions}

Many new treatment modalities have emerged with potentially more effective drugs, yet the side effects of these seem acceptable in most instances. In principle, immunosuppressive drugs are selected or designed purely to suppress lymphocyte function and inflammatory reactions, irrespective of the organ transplanted. However, in most countries drug regulatory agencies insist on controlled clinical trials for each type of transplantation separately before such a drug can be registered for use. This means that, in the case of lung transplantation, registration of these drugs comes last because of the smaller number of lung transplants performed. For example, in the Eurotransplant area of Germany, Austria, the Netherlands, Belgium and Luxembourg 3117 kidney transplants and over 1000 liver transplants were performed in 1997 compared with 158 (double or single) lung transplants. ${ }^{44}$ Lung transplant recipients are far more frequently affected by rejection than patients with other organ transplants and are at greater risk for chronic transplant dysfunction and ultimately graft loss. It is clear that they are in even greater need of more potent immunosuppressant drugs than other organ recipients. Since there is no alternative, their lives depend on keeping the graft viable for as long as possible by any means.

Lung transplantation has reached its present status mainly by developments in surgery, patient selection, and the availability of potent first generation $\mathrm{T}$ cell immunosuppressive drugs. However, further developments in lung transplantation will depend on three levels of commitment. Firstly, lung transplant physicians, committed to providing the best medical care available, are inherently inclined to use new drugs initially in rescue situations. If newer immunosuppressive drugs that are not registered for use in lung transplantation are deemed necessary, these drugs should not be withheld. Whenever possible these drugs should be given as part of a multicentre trial to establish their efficacy. Secondly, it is obvious that, despite the smaller numbers of lung transplant recipients, the frequency of chronic transplant dysfunction considerably exceeds that of other major transplant activities. For this reason the involvement of pharmaceutical companies in conducting clinical trials in lung transplantation should be encouraged early after the introduction of a new drug. Lastly, drug regulatory authorities should grant temporary registration for the use in lung transplantation of those drugs that are of proven benefit in other forms of solid organ transplantation before clinical trials in lung transplantation have been formally finalised.
1 Theodore J, Lewison N. Lung transplantation comes of age. N Engl f Med 1990;332:772

2 Sibley RK, Berry GJ, Tazelaar HD, et al. The role of transbronchial biopsies in the management of lung transplant recipients. F Heart Lung Transplant 1993;12:308-24.

3 Guilinger RA, Paradis IL, Dauber JH, et al. The importance of bronchoscopy with transbronchial biopsy and bronchoalveolar lavage in the management of lung transplant recipients. Am $\mathcal{F}$ Respir Crit Care Med 1995;152: 2037-43

4 Griffith BP, Bando K, Hardesty RL, et al. A prospective randomized trial of FK506 versus cyclosporine after human pulmonary transplantation. Transplantation 1994;57:84851 .

5 Girgis RE, Tu I, Berry GJ, et al. Risk factors for the development of obliterative bronchiolitis after lung transplantation. 7 Heart Lung Transplant 1996;15:1200-8.

6 Sarris GE, Smith JA, Shumway NE, et al. Long-term results of combined heart-lung transplantation: the Stanford experience. F Heart Lung Transplant 1994;13:940-9.

7 Bando K, Paradis IL, Similo S, et al. Obliterative bronchiolitis after lung and heart-lung transplantation. An analysis of risk factors and management. $\mathcal{F}$ Thorac Cardiovasc Surg 1995;110:4-13.

8 Scott JP, Higenbottam TW, Sharples LD, et al. Risk factors for obliterative bronchiolitis in heart-lung recipients. Transplantation 1991;51:813-7.

plantation 1991;51:813-7.
9 Kelly K, Hertz MI. Obliterative bronchiolitis. Clin Chest Kelly K, Hertz MI. O

10 Lipsky JJ. Mycophenolate mofetil. Lancet 1996;348:1357-9.

11 Bullingham R, Monroe S, Nicholls A, et al. Pharmacokinetics and bioavailability of mycophenolate mofetil in healthy subjects after single-dose oral and intravenous administration. F Clin Pharmacol 1996;36:315-24.

12 Epinette WW, Parker CM, Jones EL, et al. Mycophenolic acid for psoriasis. A review of pharmacology, longterm efficacy, and safety. 7 Am Acad Dermatol 1987;17: 962-71.

13 Sollinger HW. Mycophenolate mofetil for the prevention of acute rejection in primary cadaveric renal allograft acute rejection in primary cadaveric renal allograft Study Group. Transplantation 1995;60:225-32.

14 European Mycophenolate Mofetil Cooperative Study Group. Placebo-controlled study of mycophenolate mofetil combined with cyclosporin and corticosteroids for prevention of acute rejection. Lancet 1995;345:1321-5.

15 The Tricontinental Mycophenolate Mofetil Renal Transplantation Study Group. A blinded, randomized clinical trial of mycophenolate mofetil for the prevention of acute rejection in cadaveric renal transplantation. Transplantation 1996;61:1029-37.

16 The Mycophenolate Mofetil Renal Refractory Rejection Study Group. Mycophenolate mofetil for the treatment of refractory, acute, cellular renal transplant rejection. Transplantation 1996;61:722-9.

17 Ross DJ, Waters PF, Levine M, et al. Mycophenolate mofetil versus azathioprine immunosuppressive regimens after ung transplantation: preliminary experience. F Heart Lung Transplant 1998;17:768-74

18 Goto T, Kino T, Hatanaka H, et al. Discovery of FK-506, a novel immunosuppressant isolated from Streptomyces tsukubaensis. Transplant Proc 1987;19:4-8

19 Starzl TE, Donner A, Eliasziw M, et al. Randomised trialomania? The multicentre liver transplant trials of tacrolimus. Lancet 1995;346:1346-50.

20 The US Multicenter FK506 Liver Study Group. A comparison of tacrolimus (FK506) and cyclosporine for immunosuppression in liver transplantation. $N$ Engl $7 \mathrm{Med}$ 1994;331:1110-5.

21 European FK506 Multicentre Liver Study Group. Randomised trial comparing tacrolimus (FK506) and cyclosporin in prevention of liver allograft rejection. Lancet 1994;344:423-8.

22 Keenan RJ, Konishi H, Kawai A, et al. Clinical trial of tacrolimus versus cyclosporine in lung transplantation. Ann Thorac Surg 1995;60:580-4.

23 Kesten S, Chaparro C, Scavuzzo M, et al. Tacrolimus as rescue therapy for bronchiolitis obliterans syndrome. 7 Heart Lung Transplant 1997;16:905-912.

24 Ross DJ, Lewis MI, Kramer M, et al. FK506 'rescue' immunosuppression for obliterative bronchiolitis after lung transplantation. Chest 1997;112:1175-9.

25 Horning NR, Lynch JP, Sundaresan SR, et al. Tacrolimus therapy for persistent or recurrent acute rejection after lung therapy for persistent or recurrent acute rejection after lung

26 Sehgal SN, Molnar-Kimber K, Ocain TD, et al. Rapamycin: a novel immunosuppressive macrolide. Med Res Rev 1994; 14:1-22.

27 Cao W, Mohacsi P, Shorthouse R, et al. Effects of rapamycin on growth factor-stimulated vascular smooth muscle cell DNA synthesis. Inhibition of basic fibroblast growth factor and platelet-derived growth factor action and antagonism of rapamycin by FK506. Transplantation 1995;59:390-5

28 Gregory CR, Huang X, Pratt RE, et al. Treatment with rapamycin and mycophenolic acid reduces arterial intimal thickening produced by mechanical injury and allows endothelial replacement. Transplantation 1995;59: 655-61.

29 Akselband Y, Harding MW, Nelson PA. Rapamycin inhibits spontaneous and fibroblast growth factor beta-stimulated proliferation of endothelial cells and fibroblasts. Transplant Proc 1991;23:2833-6. 
30 Reichenspurner H, Soni V, Nitschke M, et al. Obliterative airway disease after heterotopic tracheal xenotransplantation: pathogenesis and prevention using new immunosuppressive agents. Transplantation 1997;64:373-83.

31 Fahrni JA, Berry GJ, Morris RE, et al. Rapamycin inhibits development of obliterative airway disease in a murine heterotopic airway transplant model. Transplantation 1997;63: 533-7.

32 Kelly PA, Gruber SA, Behbod F, et al. Sirolimus, a new, potent immunosuppressive agent. Pharmacotherapy 1997 17:1148-56.

33 The Rapamune Global Study Group A randomized placebo-controlled trial of Rapamune in primary renal allograft recipients. XVII World Congress of the Transplantation Society, Montreal 1998, abstract 426.

34 Backman L, Groth CG, Morales JM, et al. Rapamune (rapamycin) versus cyclosporine in a triple-drug regimen for the prevention of acute renal allograft rejection: one year results of a randomized phase II trial. XVII World Congress of the Transplantation Society, Montreal 1998, abstract 427.

35 Bonnefoy-Berard N, Revillard JP. Mechanisms of immunoBonnefoy-Berard N, Revilard JP. Mechanisms of immuno-
suppression induced by antithymocyte globulins and suppression induced by antithymocyte globulin

36 Nashan B, Moore R, Amlot P, et al. Randomised trial of basiliximab versus placebo for control of acute cellular rejection in renal allograft recipients. Lancet 1997;350 $1193-8$.
37 Vincenti F, Kirkman R, Light S, et al. Interleukin-2-receptor blockade with daclizumab to prevent acute rejection in renal transplantation. Daclizumab Triple Therapy Study

38 Lee HA, Slapak M, Raman GV, et al. Mizoribine as an alternative to azathioprine in triple therapy immunosuppressant regimens in cadaveric renal transplantation: two successive studies. Transplant Proc 1995;27:1050-1.

39 Takao M, Higenbottam TW, Audley T, et al. Effects of inhaled nebulized steroids (budesonide) on acute and chronic lung function in heart-lung transplant patients. Transplant Proc 1995;27:1284-5.

40 Speich R, Boehler A, Russi EW, et al. A case report of a double-blind, randomized trial of inhaled steroids in a patient with lung transplant bronchiolitis obliterans. Respiration 1997;64:375-80.

41 Iacono AT, Keenan RJ, Duncan SR, et al. Aerosolized cyclosporine in lung recipients with refractory chronic cyclosporine in lung recipients with refractory chronic

42 Iacono AT, Smaldone GC, Keenan RJ, et al. Dose-related reversal of acute lung rejection by aerosolized cyclosporine. Am $\mathcal{7}$ Respir Crit Care Med 1997; 155:1690-8.

43 Keenan RJ, Iacono A, Dauber JH, et al. Treatment of refractory acute allograft rejection with aerosolized cyclosporine in lung transplant recipients. F Thorac Cardiovasc Surg 1997;113:335-40.

44 Eurotransplant Newsletter 1998;145. 